\title{
RMetS
}

\section{Influence of airborne Doppler wind lidar profiles near Typhoon Sinlaku on ECMWF and NOGAPS forecasts}

\author{
M. Weissmann, ${ }^{\mathrm{a}, \mathrm{b} *}$ R. H. Langland, ${ }^{\mathrm{c} \dagger}$ C. Cardinali, ${ }^{\mathrm{d}}$ P. M. Pauley ${ }^{\mathrm{c} \dagger}$ and S. Rahm ${ }^{\mathrm{a}}$ \\ ${ }^{a}$ Deutsches Zentrum für Luft- und Raumfahrt, Institut für Physik der Atmosphäre, Oberpfaffenhofen, Germany \\ ${ }^{\mathrm{b}}$ Present address: Meteorologisches Institut, Ludwig-Maximilians-Universität, Munich, Germany \\ ${ }^{\mathrm{c}}$ Naval Research Laboratory, Monterey, California, USA \\ ${ }^{\mathrm{d}}$ European Centre for Medium-Range Weather Forecasts, Reading, UK \\ ${ }^{\star}$ Correspondence to: M. Weissmann, Ludwig-Maximilians-Universität, Meteorologisches Institut, Theresienstr. 37, \\ Munich D-80333, Germany. E-mail: martin.weissmann@lmu.de \\ ${ }^{\dagger}$ The contributions of these authors to this article were prepared as part of their official duties as United States Federal \\ Government employees.
}

\begin{abstract}
A set of about 2500 Doppler wind lidar (DWL) profiles was measured by the DLR Falcon aircraft during the life cycle of Typhoon Sinlaku in the western North Pacific as part of the THORPEX Pacific Asian Regional Campaign (T-PARC) 2008. These DWL profiles were assimilated in the global models of the European Centre for Medium-Range Weather Forecasts (ECMWF) and the Naval Research Laboratory (NRL). The beneficial impact of DWL observations is demonstrated with data denial experiments of both models and differences of the observation impact on analyses and forecasts are analysed. Additionally, the impact is quantified using the adjoint observation impact calculation. These calculations confirm the beneficial impact of DWL observations. The total relative contribution of DWL observations is about twice as high in the NRL system compared to the ECMWF system, which may be due to the lower number of satellite observations assimilated in the NRL system. In the NRL system, the DWL impact per observation is higher than that of other wind observations, whereas in the ECMWF system the DWL impact per observations is similar to other aircraft observations and lower than that of radiosondes. The results confirm preceding studies assimilating airborne DWL observations in numerical weather prediction models and underline the high expectations for future space-borne DWL instruments. Copyright (c) 2011 Royal Meteorological Society
\end{abstract}

Key Words: DWL; observation impact; data assimilation; THORPEX; T-PARC

Received 4 April 2011; Revised 21 June 2011; Accepted 5 July 2011; Published online in Wiley Online Library 11 August 2011

Citation: Weissmann M, Langland RH, Cardinali C, Pauley PM, Rahm S. 2011. Influence of airborne Doppler wind lidar profiles near Typhoon Sinlaku on ECMWF and NOGAPS forecasts. Q. J. R. Meteorol. Soc. 138: 118-130. DOI:10.1002/qj.896

\section{Introduction}

The number of observations used in data assimilation systems of major numerical weather prediction (NWP) centres has increased drastically over the last decade, e.g. by about a factor of 10 at the European Centre for Medium-Range Weather Forecasts (ECMWF) (Isaksen, 2010). However, most of the additional observations are radiances observed by passive satellite instruments that mainly provide information about the mass and humidity fields. The amount of wind observations has been nearly stagnant, with the exception of atmospheric motion vectors (AMVs) derived from satellite imagery. These AMVs, however, exhibit horizontal error correlation lengths of up to $800 \mathrm{~km}$ (Bormann et al., 2003), which are mainly due to uncertainties of their height assignment (Velden 
and Bedka, 2009). For this reason, AMVs are usually either thinned rigorously or their errors are inflated for data assimilation systems of global NWP models. Conventional wind observations are concentrated on the Northern Hemisphere's continents and aircraft corridors in the upper troposphere and lower stratosphere, while large parts of the globe are unobserved. According to Emanuel et al. (1997), this relative paucity of routine observations in remote regions is one of the most significant impediments to progress in weather forecasting.

In such a framework, Doppler wind lidar (DWL) offers a promising opportunity to observe the wind field either globally, with a polar-orbiting satellite (Stoffelen et al., 2005; ESA, 2008), or regionally, if mounted on aircraft (Weissmann etal., 2005). The launch of the first DWL in space, the Atmospheric Dynamics Mission Aeolus instrument (ADM-Aeolus) of the European Space Agency (ESA), is still a few years in the future. Airborne DWL in contrast, has been deployed during recent field campaigns, e.g. the Atlantic THORPEX Regional Campaign 2003 (A-TReC) and the THORPEX Pacific Asian Regional Campaign (T-PARC) 2008. The assimilation of airborne DWL observations from eight flights over the North Atlantic during A-TReC in the ECMWF Integrated Forecast System (IFS) documented a high analysis influence of DWL observations due to their low observational error. In addition, the observations led to a significant reduction of the mean geopotential 2- to 4-day forecast error over Europe by about 3\% in a 2 -week period (Weissmann and Cardinali, 2007). A recent case study with DWL observations made near Typhoon Nuri during T-PARC also revealed a positive influence of DWL observations on typhoon track and intensity forecasts of the Weather and Research Forecasting (WRF) model (Pu et al., 2010).

The goal of this paper is to validate the results of Weissmann and Cardinali (2007) and $\mathrm{Pu}$ et al. (2010) with a larger DWL dataset, to quantify the impact of DWL observations on NWP forecasts, and to gain further insight into how DWL observations act in global data assimilation systems. The number of observations is one crucial ingredient in the confidence of studies using data denial experiments. T-PARC provides about 2500 wind profiles within 11 days during the life cycle of Typhoon Sinlaku observed by the $2 \mu \mathrm{m}$ scanning coherent DWL onboard the DLR Falcon aircraft. This is about twice as many DWL observations as during the A-TReC campaign. Two different assimilation and modelling systems - the ECMWF IFS and the NRL Navy Operational Global Atmospheric Prediction System (NOGAPS) - are used to quantify and compare the observation influence. The adjoint observation impact (Langland and Baker, 2004; Cardinali, 2009; Gelaro et al., 2010), which provides the reduction of forecast error by every assimilated observation, was calculated in both systems in addition to data denial experiments.

The paper is outlined as follows. Section 2 describes the observations, synoptic environment, modelling systems and the adjoint observation impact calculation. Section 3 presents the assimilation statistics of the observations and compares the analysis and forecast increments in both modelling systems. The DWL influence on forecast errors is then quantified in section 4 using a verification with typhoon best-track data, a verification with model analyses and adjoint observation impact calculations. Conclusions are presented in section 5 .

\section{Description of observations and modelling systems}

\subsection{Falcon flights during T-PARC}

The summer component of the multinational T-PARC field campaign was conducted in August to October 2008 in the western North Pacific. The campaign aimed to improve typhoon track and intensity forecasts by targeted observations and to investigate the extratropical transition of tropical cyclones (TCs) and their downstream impact in midlatitudes.* The DLR Falcon 20 aircraft performed 25 research flights during the campaign to address both objectives. Eleven of them - the flights during the lifetime of Typhoon Sinlaku in the period 11-21 September 2008 - are used in the present study (Figure 1). The flights before Sinlaku did not provide DWL observations for technical reasons. Observations for Typhoon Jangmi in the period 28 September to 1 October were not used owing to the gap between Sinlaku and Jangmi observations of about one week and computational restrictions.

Typhoon Sinlaku formed as a tropical depression east of the Philippines on 8 September 2008, intensified to a typhoon on 9 September, underwent rapid intensification and reached a minimum pressure of $935 \mathrm{hPa}$ by $1800 \mathrm{UTC}$ on 10 September. The first Falcon mission for Sinlaku on 11 September covered the northern and eastern environment of Sinlaku with a double flight from Atsugi, Japan (near Tokyo) to the island of Okinawa and back to Atsugi (Figure 1). These flights intended to sample the edge of the subtropical high to the east of Sinlaku and the interface to the jet stream to the north. In the following days, Sinlaku slowly meandered towards Taiwan, which was hit heavily on 14 September. A flight on 14 September covered the upper-level jet to the north of Sinlaku that was indicated to be sensitive for the track forecast by singular vector calculations. Sinlaku recurved in the East China Sea on 15 September and accelerated its movement thereafter. The next mission was conducted on 16 September - a similar double flight for the purpose of targeted observations in the environment of Sinlaku as on 11 September. The intensity of Sinlaku decreased to a tropical depression after hitting Taiwan, but in contrast to most forecasts it reorganized and reached typhoon intensity again south of Japan on 18 September. The Falcon mission on 17, 18, 19 and 21 September focused on the interaction of Sinlaku with the midlatitude jet stream and either went into the environment of Sinlaku (particularly into the outflow region) or into the upper-level jet to the northwest. During the last flight on 21 September, the storm had nearly dissipated east of Japan. Sinlaku interacted with the midlatitude jet (Harr et al., 2010), but it neither transformed into a significant extratropical system nor caused a strong downstream development.

\subsection{DWL system and observations}

During T-PARC, the DLR Falcon was equipped with a scanning DWL, a dropsonde system and a differential absorption lidar (DIAL) for water vapour. DIAL observations are not used in this study and dropsondes only for the comparison of the DWL and dropsonde impact on typhoon tracks and in the ECMWF verification analysis.

*T-PARC website: http://www.eol.ucar.edu/projects/t-parc/ 

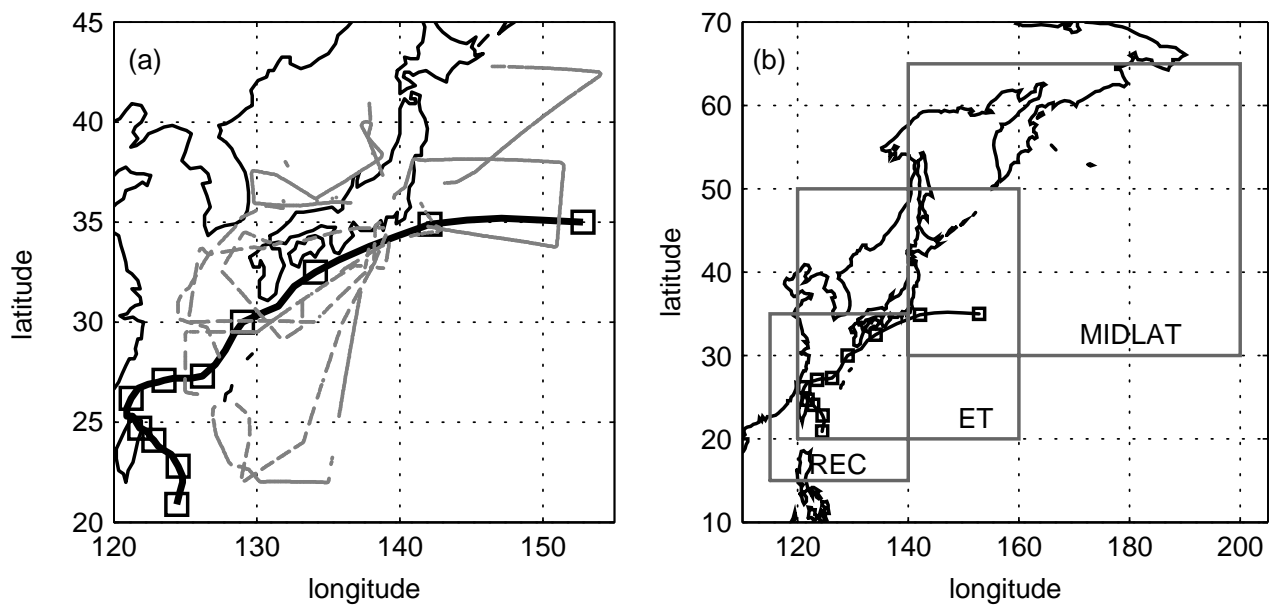

Figure 1. (a) Location of DWL profiles (grey) and Typhoon Sinlaku (black line) according to the JMA best-track for the period 11-21 September 2008 with squared markers at 0000 UTC, respectively. (b) Boxes used for the verification of forecasts with model analyses from southwest to northeast: region of Sinlaku recurvature (REC), region of extratropical transition and jet interaction (ET) and region of Sinlaku midlatitude influence (MIDLAT).

The DWL system measures wind profiles beneath the aircraft using the Velocity-Azimuth Display (VAD) technique (Smalikho, 2003). The instrument performs a conical step-and-stare scan around the vertical axis at $20^{\circ}$ off nadir that, combined with the movement of the aircraft, results in a cycloid scan pattern. During T-PARC, the lineof-sight (LOS) observations from one scanner revolution, i.e. 24 LOS observations, were used for every wind profile. This results in wind profiles with a horizontal resolution of about $5 \mathrm{~km}$ and a vertical resolution of $100 \mathrm{~m}$. A more detailed description of the system can be found in Weissmann et al. (2005).

The principle of Doppler lidar is in many ways similar to that of Doppler radars, except that a lidar emits pulses of laser light instead of radio waves (Grund et al., 2001). The Falcon DWL emits a signal at a wavelength of $2.02254 \mu \mathrm{m}$. Thus it requires sufficient aerosols with a diameter similar to the emitted wavelength to receive a backscatter signal to determine the Doppler shift and consequently the LOS velocity and horizontal wind vector. The intensity of the received signal decreases with the squared distance of the observed volume from the aircraft and the signal cannot penetrate optically thick clouds. As a result, the DWL can provide full wind profiles from the aircraft to the ground under beneficial conditions (e.g. no clouds, high aerosol concentrations and high humidity, which causes a swelling of aerosols), but often the profiles are intermittent. On average, every DWL wind profile during T-PARC provided wind information on about $2500 \mathrm{~m}$ of the vertical profile similar to the observations during A-TReC (Weissmann et al., 2005). This means that $20-25 \%$ of the vertical profile contains wind information. The highest coverage of DWL observations occurred between 250 and $300 \mathrm{hPa}(1-2 \mathrm{~km}$ beneath the aircraft flight level) and the second highest coverage in the atmospheric boundary layer due to higher aerosol concentrations, whereas the coverage was particularly low between 500 and $800 \mathrm{hPa}$.

The whole T-PARC DWL dataset consists of more than 4000 wind profiles; about 2500 of them (62 689 observations of horizontal wind) are in the Sinlaku period and are used for this study.

\subsection{ECMWF IFS}

The spring 2009 version (cycle 35r2) of the ECMWF IFS, with a resolution of $\sim 25 \mathrm{~km}$ (T799) and 91 vertical levels, was used for the experiments (Table I). Two experiments - one without DWL observations (ECCTR) and one with DWL observations (ECDWL) - were conducted for the period 11-21 September 2008, which covers all Falcon DWL observations during the life cycle of Typhoon Sinlaku. The experiments were cycled, i.e. the additional observations modified the ECDWL first-guess field and consequently also those ECDWL analyses without DWL observations. Dropsondes were not used in these two experiments to have a clear signal of the assimilation of DWL observations since many dropsondes were collocated with DWL profiles. Besides dropsondes, all the observations operationally received were assimilated in the ECMWF model ( $\sim 18$ million per day). An independent experiment (ECDROP) including no DWL observations but dropsondes (about 300 soundings in the period and area of Sinlaku) is used for the forecast verification and for the comparison of DWL and dropsonde impact on ECMWF typhoon track forecasts.

The experiments were performed with a $12 \mathrm{~h}$ window 4D-Var data assimilation system (Rabier et al., 2000). The innovations and residuals (differences of observations to the first-guess and analysis fields in observations space, respectively) are calculated at full resolution. The $4 \mathrm{D}$ Var incremental approach counts an outer loop at full forecast resolution and three inner loops at T255, T159 and T95, respectively. No vortex relocation or synthetic vortex observations are used for TCs in the ECMWF modelling system.

Similar to the experiments of Weissmann and Cardinali (2007), the error variance assigned to DWL observation is $1.5 \mathrm{~m} \mathrm{~s}^{-1}$. At the beginning of the assimilation procedure, the observations were thinned to $0.5675^{\circ}$ latitude/longitude ( $\sim 63 \mathrm{~km}$ ) horizontally and to 35 levels beneath $200 \mathrm{hPa}$ in the vertical. In the same way as for conventional wind data, a first-guess check (FG-check) eliminates all observations whose innovation squared exceeds five times the sum of the expectations (observation and background error) squared. After the FG-check, a variational quality control (VarQC) is performed in the minimization procedure (Andersson and 
Table I. Comparison of ECMWF and NOGAPS modelling system.

\begin{tabular}{llcclcccc}
\hline & $\begin{array}{l}\text { Model } \\
\text { resolution }\end{array}$ & $\begin{array}{c}\text { Vertical } \\
\text { model } \\
\text { levels }\end{array}$ & $\begin{array}{c}\text { Number of } \\
\text { DWL } \\
\text { observations }\end{array}$ & $\begin{array}{l}\text { Number of } \\
\text { assimilated } \\
\text { DWL } \\
\text { observations }\end{array}$ & $\begin{array}{c}\text { Lidar } \\
\text { horizontal } \\
\text { resolution }\end{array}$ & $\begin{array}{c}\text { Lidar } \\
\text { vertical } \\
\text { resolution }\end{array}$ & $\begin{array}{c}\text { QC flags } \\
\text { FG (VarQC) }\end{array}$ & $\begin{array}{c}\text { Assigned } \\
\text { error } \\
\left(\mathrm{m} \mathrm{s}^{-1}\right)\end{array}$ \\
\hline ECMWF & $\begin{array}{l}\text { T799 } \\
(\sim 25 \mathrm{~km})\end{array}$ & 91 & 125378 & 9578 & $\sim 5 \mathrm{~km}$ & $100 \mathrm{~m}$ & $\begin{array}{c}236 \\
(150)\end{array}$ & 1.5 \\
NOGAPS & $\begin{array}{l}\text { T239 } \\
(\sim 55 \mathrm{~km})\end{array}$ & 42 & 125378 & 4368 & $\begin{array}{c}1.5^{\circ} \\
\text { lat./long. }\end{array}$ & $400 \mathrm{~m}$ & $\begin{array}{c}0 \\
(0)\end{array}$ & 1.8 \\
\hline
\end{tabular}

Järvinen, 1999) to decrease the weight of remaining doubtful observations.

For a better comparison to lower-resolution NOGAPS experiments, the forecast verification and track calculation is performed with model fields reduced to a horizontal resolution of $1^{\circ}$ latitude/longitude. Typhoon tracks and intensity are determined by searching the sub-grid minimum of sea-level pressure as in Harnisch and Weismann (2010).

\subsection{NRL NAVDAS and NOGAPS}

The NRL Atmospheric Variational Data Assimilation System - Accelerated Representer (NAVDAS-AR; Rosmond and $\mathrm{Xu}, 2006$ ) and the NOGAPS forecast model, with a horizontal resolution of $\sim 55 \mathrm{~km}$ (T239) and 42 levels in the vertical, were used (Table I). Two cycled experiments were conducted for the period 11-21 September 2008, one without DWL observations (NRLCTR) and one with DWL observations (NRLDWL). All observations used in the operational version of NAVDAS-AR ( $\sim 3$ million per day) with the exception of dropsondes were assimilated in both experiments. NAVDAS-AR also assimilates synthetic vortex (bogus) observations based on the TC intensity estimate from the official warning centres (Goerss and Jeffries, 1994) to receive a realistic intensity of TCs. A single-cycle sensitivity experiment with DWL, but no bogus observations (NRLNBG), was performed for 1200 UTC on 11 September.

NOGAPS (Hogan and Rosmond, 1991) is a primitive equation spectral model with a complete set of moist physics, including an Emanuel cumulus parametrization (Hogan and Pauley, 2006). NAVDAS-AR is a $6 \mathrm{~h}$ window 4D-Var data assimilation system (Rosmond and Xu, 2006). The minimization of the cost function (i.e. calculation of analysis increments) is performed at a reduced horizontal resolution of $\sim 110 \mathrm{~km}$ (T119).

A superobbing procedure was applied to the DWL observations to average values within $1.5^{\circ}$ latitude/longitude horizontally and $400 \mathrm{~m}$ vertically. This superobbing scheme also includes a quality check based on the consistency of observations within the averaging volume. A constant error of $1.8 \mathrm{~m} \mathrm{~s}^{-1}$ was assigned to the DWL observations, the same error as to low-level radiosonde wind observations, which represent the most accurate operational wind observation.

The operational NRL TC tracker is used to define the position and intensity of the storm and $1^{\circ}$ latitude/longitude fields are used for the forecast verification with model analyses (as for ECMWF experiments).

\subsection{Comparison of DWL assimilation procedure}

The assimilation of DWL observations differs in the two modelling systems. First, there are differences between the data assimilation and modelling systems themselves; e.g. the horizontal resolution of ECMWF is more than twice as high (Table I) and the number of assimilated operational observations is higher by roughly a factor of six due to the more extensive use of radiances at ECMWF. Secondly, the observations are treated differently since the performed experiments use quality control and assimilation procedures as found in the existing operational systems of ECMWF and NAVDAS-AR.

In NAVDAS-AR, a superobbing scheme is applied to the DWL observations before the assimilation, while a thinning procedure is used in the ECMWF assimilation system. This means that the observations in NAVDAS-AR represent a $1.5^{\circ} / 400 \mathrm{~m}$ average in comparison to $5 \mathrm{~km} / 100 \mathrm{~m}$ in the ECMWF system. The superobbing also includes an additional quality check.

The assigned observation error variance of $1.8 \mathrm{~m} \mathrm{~s}^{-1}$ for DWL observations in NAVDAS-AR is slightly higher than the value of $1.5 \mathrm{~m} \mathrm{~s}^{-1}$ used in the ECMWF system. Both estimates of observation error variance are within the uncertainty of the observation accuracy and Weissmann and Cardinali (2007) showed that differences of this magnitude should have no significant influence on forecast outcome. The superobbing/thinning also influenced the number of assimilated observations, which is about two times higher in the ECMWF system.

However, because of the superobs assimilation procedure in the NOGAPS system, all DWL observations contribute to the analysis, while $92 \%$ of the observations are not used in the ECMWF system because of thinning. Overall, the DWL observations are given slightly more weight in the ECMWF analyses due to the larger number of assimilated observations and lower assumed errors.

\subsection{NOGAPS adjoint observation impact}

Observation impact on forecast error in NOGAPS is calculated using the adjoint-based procedure developed in Langland and Baker (2004). The $24 \mathrm{~h}$ global forecast error is computed in terms of a moist total energy norm from the surface up to about $150 \mathrm{hPa}$. Each observation is therefore either reducing or increasing the forecast error. The observation impact may be gathered according to the observation type, observation variable, pressure level and geographic region. For the observation impact calculation, the adjoint of NOGAPS is run at T239, which is the same resolution as the forecast model in this experiment. The 
adjoint of NAVDAS-AR is run at the same resolution as the regular data assimilation (T119).

Since 2006, the observation impact has been routinely computed at NRL Fleet Numerical Meteorology and Oceanography Center (NRL-FNMOC) and is used for quality control, satellite channel selection and evaluation of new observation types. A comparison of adjoint-based observation impact in three different forecast systems is described by Gelaro et al. (2010).

\subsection{ECMWF adjoint observation impact}

The ECMWF adjoint-sensitivity tool computes the observation impact using a global dry total energy norm from the surface up to $0.1 \mathrm{hPa}$ as a measure of $24 \mathrm{~h}$ forecast error and a full representation of moist processes in the adjoint calculation (see Cardinali, 2009, for more details). For the observation impact calculation, the resolution of the analysis, its adjoint and the adjoint forecast model is T255, while the forecast resolution is T799.

At the implementation of the observation impact calculation at ECMWF, several tests have been performed to assess the different approximations applied in the computation of the forecast sensitivity to observations. In particular, one of the tests estimated the error of considering only one outer loop instead of the three loops computed in the analysis. The error was globally less than $1 \%$ when all observations were assimilated.

The forecast error cost function extends from the surface to about $150 \mathrm{hPa}$ in NOGAPS and to $0.1 \mathrm{hPa}$ in ECMWF. In terms of observation impact results, this difference in cost function definition will (a) increase the magnitude of forecast error in the ECMWF results since more of the atmosphere is included, and (b) increase the proportion of observation impact due to stratospheric observations since those observations are most likely to affect forecast error in pressure levels between 0.1 and $150 \mathrm{hPa}$. Thus, it can also be inferred that the relative impact of tropospheric observations (including DWL) is likely reduced in the ECMWF results, compared to the NOGAPS results.

\section{DWL influence}

\subsection{Innovations}

The distribution of innovations (differences between observations and model first-guess) in both systems is fairly symmetric and resembles a normal distribution (Figure 2). The magnitude of the root mean square (RMS) of the innovations is consistent with the values described in Weissmann and Cardinali (2007). Despite differences of the assimilation procedure in the two models (section 2.5), the RMS of the innovation of all assimilated DWL observations is about $2.85 \mathrm{~m} \mathrm{~s}^{-1}$ in both systems (Figure 3). The NRL superobbing is expected to reduce the observation and representativeness errors, while the higher ECMWF resolution and large amount of other observations assimilated are expected to lead to a more accurate analysis and first-guess field. During the first flights near Typhoon Sinlaku, the ECMWF first-guess has a more realistic storm structure, leading to smaller innovations. However, on the two flights into the jet stream north of Sinlaku over the Sea of Japan on 18 September and east of northern Japan on 19 September, the ECMWF innovations are larger.
The histogram of the innovations in both systems shows that ECMWF has a larger fraction of innovations near zero, which again shows a better first-guess on average, but also a larger fraction of outliers, which seem to be better handled by the superobbing procedure in the NRL system (Figure 2).

\subsection{Analysis and forecast increments}

In both systems, the residuals (difference between observations and analysis) of DWL observations are clearly lower than the innovations, proving that the observations were successfully assimilated (Figures 2 and 3). Quality control flags and the residuals of other observations do not show a systematic difference in the use of other operational observations (not shown).

According to the lower assigned error and the larger amount of DWL observations assimilated, ECMWF also shows a larger mean analysis increment and a lower RMS of the residuals of all assimilated DWL observations. This also implies that the larger amount of satellite observations assimilated in the ECMWF system does not conflict with the assimilation of DWL observations.

Figure 4 shows the mean absolute difference of geopotential height forecasts with and without DWL observations averaged over the whole period and the area ET (see Figure 1) for ECMWF and NRL, respectively. The difference of $1000 \mathrm{hPa}$ geopotential height is very similar in both systems up to a forecast lead time of $72 \mathrm{~h}$. The difference of $500 \mathrm{hPa}$ geopotential height is lower in the ECMWF system at all time steps despite the larger weight given to DWL observations and larger analysis increments at the location and time of DWL observations. This is likely related to the larger amount of radiance data assimilated at ECMWF that constrains the mass field and through the balance relation the wind field, which reduces the influence of direct but localized wind observations. At $96 \mathrm{~h}$ lead time, ECMWF also exhibits a lower difference at $1000 \mathrm{hPa}$.

\section{DWL impact on forecast error}

\subsection{Typhoon forecasts}

ECMWF and NOGAPS track and intensity forecasts of Typhoon Sinlaku are verified with best-track data from the Japan Meteorological Agency (JMA). For comparison, the track error of an experiment with all T-PARC dropsondes performed by Weissmann et al. (2011) is also displayed (ECDROP in Figure 5(a)).

The ECMWF experiments show a reduction of the mean ECDWL track forecast error between 5 and $50 \mathrm{~km}$ compared to ECCTR (Figure 5(a)). The maximum improvement is smaller than the improvement of up to $90 \mathrm{~km}$ of ECDROP, but the mean relative improvement of $12-120 \mathrm{~h}$ track errors is $9 \%$ in ECDWL compared to $8 \%$ in ECDROP due to larger relative improvements of ECDWL at shorter lead times.

The DWL influence on the NOGAPS typhoon track and intensity forecast skill is overall neutral. Both NRLCTR and NRLDWL exhibit mean track forecast errors that are more than twice as high as ECMWF errors at lead times $12-36 \mathrm{~h}$, about $50-100 \%$ higher at lead times $48-72 \mathrm{~h}$ and of a similar magnitude as ECMWF afterwards (Figure 5). The low influence of DWL observations in NOGAPS experiments is also demonstrated by the example of track forecasts initialized at 1200 UTC on 11 September presented in 

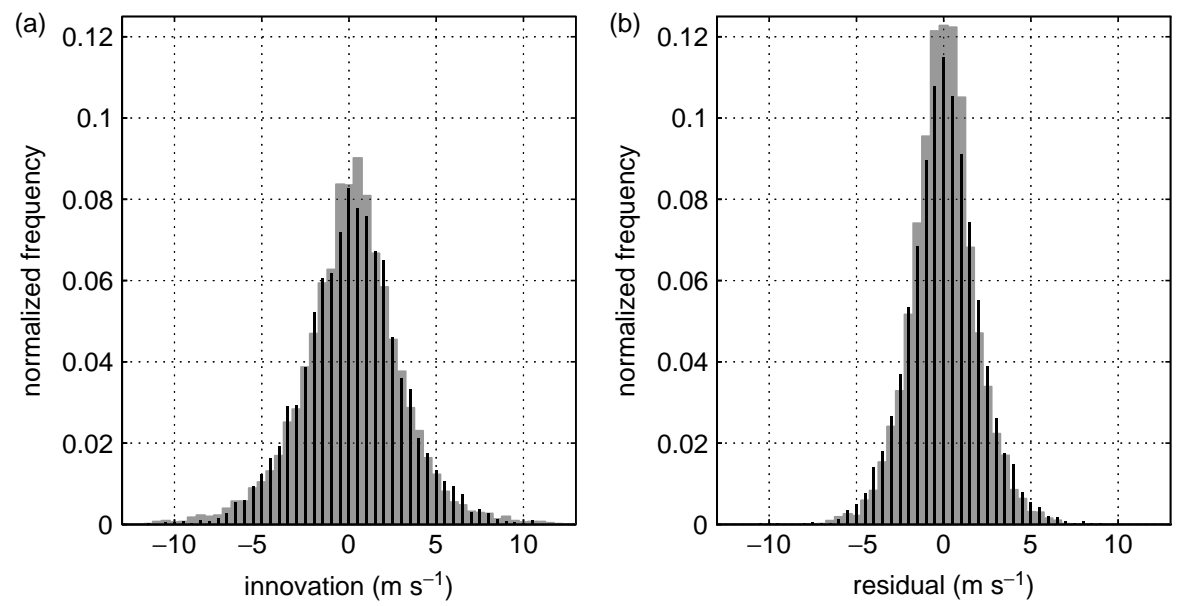

Figure 2. Normalized histogram of DWL (a) innovations and (b) residuals for all assimilated DWL observations without quality control flags in NAVDAS-AR (narrow black bars) and the ECMWF system (wide grey bars).

Figure 6(a), where NRLCTR and NRLDWL track forecasts are nearly identical up to day 2 and similar afterwards.

Figure 6(c) shows an example of bogus and DWL observations on 11 September. Bogus observations cover the whole storm and its immediate environment and they are assimilated with low assigned error variances (i.e. high weight) similar to the DWL observations that are only located to the north and east of the storm. Figure 6(a) and (b) shows a sensitivity study, where bogus observations were not used in one analysis cycle (experiment NRLNBG). A considerably improved Sinlaku track prediction with landfall on Taiwan is achieved. From a forecaster's perspective, this would be an important improvement as the timely prediction of landfall is crucial for evacuation and warning procedures. However, the bogus removal in one analysis cycle also leads to a weakening of the storm by a few $\mathrm{hPa}$ (and the weakening may even be larger with removal of the bogus in several successive analysis cycles). It seems that the inclusion of the synthetic TC bogus vortex with a horizontal extension of $10^{\circ} \times 10^{\circ}$ latitude and longitude reduces the influence of other observations assimilated in NAVDAS-AR. Ongoing studies (not shown) at NRL indicate that the TC bogus, on average, improves NOGAPS forecasts of TC intensity and track. However, a significant fraction of TC forecasts is degraded by assimilation of the synthetic vortex. Section 4.3 further investigates the influence of bogus observations using adjoint observation impact calculations.

Overall, one major issue of NOGAPS Sinlaku forecasts is that despite the assimilation of bogus data, the analysis is not able to reproduce the intensification of Sinlaku that occurred the day before the observations were first released on 11 September. Sinlaku is consistently too weak in the NOGAPS analysis and even weaker in the forecast (Figure 5(d)). Before the landfall on Taiwan, the minimum pressure of Sinlaku is nearly $50 \mathrm{hPa}$ higher than the best-track data. After the landfall, the pressure difference decreases to about $15 \mathrm{hPa}$ due to the weakening of Sinlaku by $40 \mathrm{hPa}$, but Sinlaku still appears to be too weak in all NOGAPS experiments and a few forecasts even miss the distinct circulation centre. The comparably low resolution of NOGAPS is likely one contributor to the weakness of Sinlaku in the NRL system.

ECMWF forecasts also show a minimum pressure that is $45 \mathrm{hPa}$ too high in the first analysis cycle, but the difference to the best-track data reduces to $25 \mathrm{hPa}$ on the following day, without the use of bogus data. After landfall, the storm is about $10 \mathrm{hPa}$ too weak in the ECMWF analysis - a difference that appears fairly reasonable keeping in mind the limited model resolution and the fact that the intensity was calculated with $1^{\circ}$ latitude/longitude model output. A distinct circulation centre and pressure minimum of Sinlaku can be found in all forecasts. The ECMWF forecast model tends to produce more intense TCs than the analysed ones due either to the lower analysis resolution or the background covariance matrix specification that does not properly modulate intense storms. The ECMWF Sinlaku forecast minimum pressure is up to about $10 \mathrm{hPa}$ lower than in the analysis (Figure 5). The opposite behaviour is seen for NOGAPS, where the forecast of Sinlaku is even slightly weaker than the analysis.

The influence of DWL observations on ECMWF intensity forecasts is negligible, as in NOGAPS. Observations closer to the storm might have a larger influence on intensity forecasts, but it should also be kept in mind that even at the ECMWF resolution of $25 \mathrm{~km}$ the model cannot fully resolve the typhoon.

\subsection{Forecast verification with model analyses}

Forecasts from both models are verified using an ECMWF analysis with all operational observations including dropsondes, but no DWL data (ECDROP). Three verification boxes (see Figure 1(b)) are used that cover the evolution of Sinlaku and the largest impact of the observations: the first one is focused on the recurvature of Sinlaku (REC), the second one on its extratropical transition and interaction with the midlatitude jet (ET) and the third one on its downstream impact in midlatitudes (MIDLAT).

Consistent with the track forecast improvement, ECMWF shows a comparably large reduction of the forecast error in the area REC of up to $8 \%$ at $1000 \mathrm{hPa}$ and up to $5 \%$ at $500 \mathrm{hPa}$ (Figure 7). NOGAPS forecasts also improve in this area by up to $3 \%$ and $5 \%$ at 1000 and $500 \mathrm{hPa}$, respectively, although no improvement is visible in the verification with best-track data.

Improvement is also seen in the ET area in both models, but the error reduction is not as consistent for all forecast times as in the REC area. NOGAPS forecasts improve up to $5 \%$ with the exception of $2 \%$ deterioration of $1000 \mathrm{hPa}$ fields at $24 \mathrm{~h}$ lead time and a neutral impact at $48 \mathrm{~h}$. 

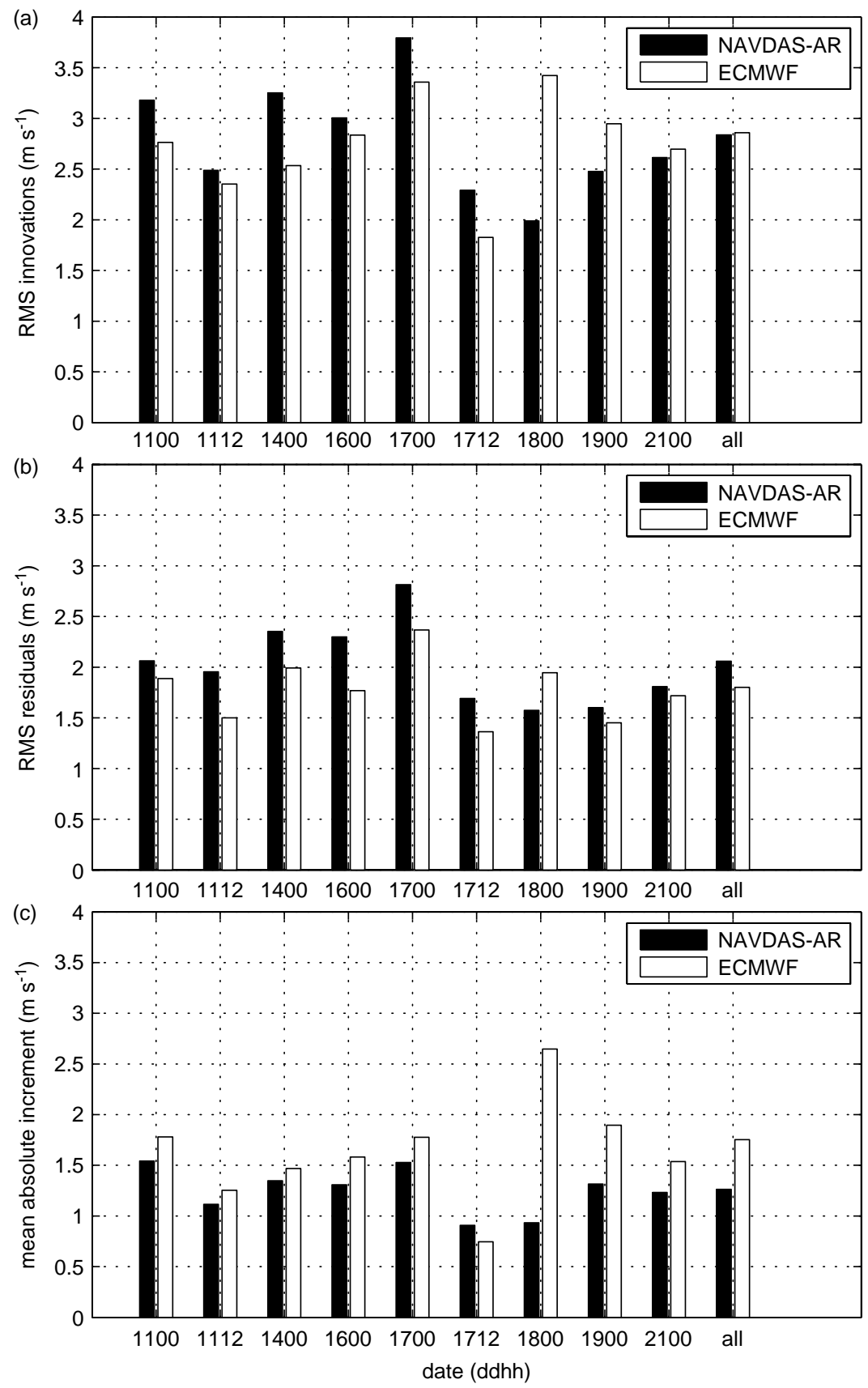

Figure 3. Assimilation statistics of DWL observations without quality control flags in NAVDAS-AR and the ECMWF system: (a) RMS of innovations; (b) RMS of residuals; and (c) mean absolute analysis increment at the position of DWL observations, i.e. mean absolute difference between innovation and residual.

ECMWF forecasts improve at all time steps except at $48 \mathrm{~h}$, where a slight deterioration appears at $1000 \mathrm{hPa}$ and a neutral impact at $500 \mathrm{hPa}$. For both the REC and the ET area, the differences are only significant for a few time steps.

NOGAPS forecasts also indicate improvement at lead times of 96 and $120 \mathrm{~h}$ downstream in the MIDLAT area at $500 \mathrm{hPa}$, whereas the impact at $1000 \mathrm{hPa}$ is neutral. ECMWF seems to deteriorate in the MIDLAT area. However, none of the ECMWF differences in the MIDLAT area is significant.

\subsection{NOGAPS observation impact using adjoint methods}

The impact of all assimilated observations on a measure of short-range forecast error can be quantified using adjoint versions of NOGAPS and NAVDAS-AR, following the method derived by Langland and Baker (2004). Here we examine the impact of observations on reduction of a moist total energy norm defined from $24 \mathrm{~h}$ forecast errors of temperature, humidity, wind and surface pressure in NOGAPS.

Results from the $24 \mathrm{~h}$ adjoint observation impact calculations for DWL observations assimilated in the area around Sinlaku generally confirm the results of the NOGAPS data denial experiments. The DWL observations are overall beneficial, with an average error reduction of $2.06 \times 10^{-5} \mathrm{~J} \mathrm{~kg}^{-1}$ per observation, a total error reduction of $0.09 \mathrm{~J} \mathrm{~kg}^{-1}$ and a fraction of $51.4 \%$ beneficial observations (Table II). Eight out of 12 analysis cycles show an overall beneficial influence of DWL observations. 


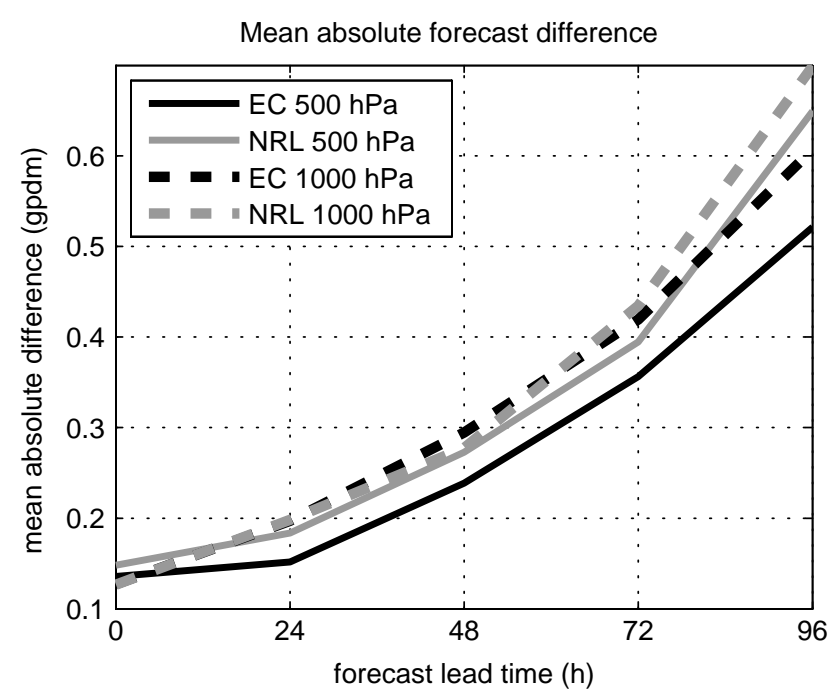

Figure 4. Mean absolute geopotential height forecast difference (gpdm) for ECMWF (black) and NRL NOGAPS (grey) experiments with and withou DWL observations averaged over the area ET shown in Figure 1 and the period 11-21 September 2008.

Consistent with the findings of Gelaro et al. (2010), the three global observation types with the largest total contribution to the reduction of $24 \mathrm{~h}$ forecast error are AMVs derived from images by geostationary satellites (AMV GEO), radiosonde observations (TEMP), and radiances observed by Advanced Microwave Sounding Unit-A (AMSU-A) instruments onboard satellites of the National Oceanic and Atmospheric Administration (Figure 8(a)). Owing to the comparably low number of observations, the global relative contribution of DWL observations to the reduction of $24 \mathrm{~h}$ forecast error is small $(\sim 0.14 \%)$, but the mean impact per DWL observation is higher than that of all other observations types, except synthetic TC bogus observations (TC Synth), Australian synthetic surface pressure observations (AUS_syn) and observations of total precipitable water derived from passive satellite instruments (WINDSAT-TPW and SSMI-TPW, Figure 8(b)). The high beneficial influence of synthetic observations on a global scale also underlines that the bogus is still required in the NOGAPS system.

In the area of the DWL observations, the largest total contribution comes from radiosondes, AMVs and aircraft observations with around or over $20 \%$ each, whereas AMSUA contributes only about $6 \%$ (Figure $8(\mathrm{c})$ ). The total relative contribution of DWL observations in the area is about $3 \%$. Again, the DWL contribution per observation is higher than that of most other observation types, but in addition to synthetic TC bogus and TPW observations also surface winds from the Advanced Scatterometer (ASCAT SFC WIND) have a higher contribution per observation in the area around Typhoon Sinlaku (Figure 8(d)).

Averaged over the same analysis cycles as the DWL impact, the bogus for Typhoon Sinlaku is also beneficial and contributes about as much to the reduction of forecast error as the DWL observations (Table II). The mean contribution per bogus observation is even four times higher than that of DWL, which is likely due to the fact that bogus observations are (a) within the TC region that is covered by very few other observations, (b) likely in a sensitive environment and (c) assimilated with fairly low errors. The observation impact calculation even shows a beneficial influence of the bogus at 1200 UTC on 11 September, although the sensitivity experiment without bogus (NRLNBG) initialized on this day leads to a much-improved track forecast compared to the experiments NRLCTR and NRLDWL (section 4.1, Figure 6). The observation impact with and without the bogus (Table III) demonstrates a strong influence of the bogus removal on the impact of other observation types. Without the bogus, all other observation types in the Sinlaku area except aircraft provide larger reduction of the $24 \mathrm{~h}$ forecast error norm. The larger impact of the other observation types more than compensates for the eliminated bogus, increasing the $24 \mathrm{~h}$ error norm reduction by $43 \%$.

\subsection{ECMWF observation impact using adjoint methods}

The observation impact in the ECMWF system is calculated similarly to the NOGAPS system (see sections 2.7 and 2.8). However, differences arise from the averaging period: ECMWF observation impact is computed for the period 11-21 September, whereas the NOGAPS impact is averaged only over the twelve 6-h assimilation cycles that contain DWL observations. The full period consists of 40 NAVDASAR $6 \mathrm{~h}$ analysis windows. The total impact of all observations in the area considered is fairly constant. Therefore, NOGAPS and ECMWF relative DWL forecast error contributions are comparable after accounting for the different number of analysis cycles, i.e. multiplying the ECMWF contribution by a factor of 3.3 to account for the fact that only $30 \%$ of the $6 \mathrm{~h}$ windows in the period contain DWL observations. In the ECMWF system, the DWL observations (Figure 9(a)) contribute about $0.5 \%$ to the reduction of forecast error with respect to all the observations in the area $20-50^{\circ} \mathrm{N}, 120-160^{\circ} \mathrm{W}$. Thus the relative DWL contribution in the ECMWF system is about half that in NOGAPS, after accounting for the different number of analysis cycles. Radiosonde and wind profiler (TEMP and PILOT) observations contribute about $30 \%$ to the reduction of forecast error, followed by aircraft observations $(\sim 17 \%)$, AMSU-A $(\sim 11 \%)$ and scatterometer surface winds (SCAT, $\sim 8 \%$ ).

The highest contribution per observation (Figure 9(b)) in the ECMWF system comes from drifting buoys, presumably because these provide quite unique surface pressure information in data-sparse regions. The contribution per observation of AMVs, synoptic surface observations (SYNOP), radiosondes and wind profilers is also comparably high. The DWL contribution per observation is similar to other aircraft observations. For comparing the mean relative contribution per observation in NOGAPS and ECMWF (Figures 8(d) and 9(b)), one needs to consider that (a) more observations are assimilated in the ECMWF analysis and (b) more cycles are used for the calculation (as described at the beginning of this section). Consequently, the number of observations is about one magnitude larger for ECMWF and the mean contribution per observation is about one magnitude smaller for ECMWF.

\section{Conclusion}

This study investigates the impact of airborne DWL observations in the ECMWF and NOGAPS global modelling systems. About 2500 additional wind profiles over the northern West Pacific were assimilated in an 11-day period during the summer phase of the T-PARC 2008 field 

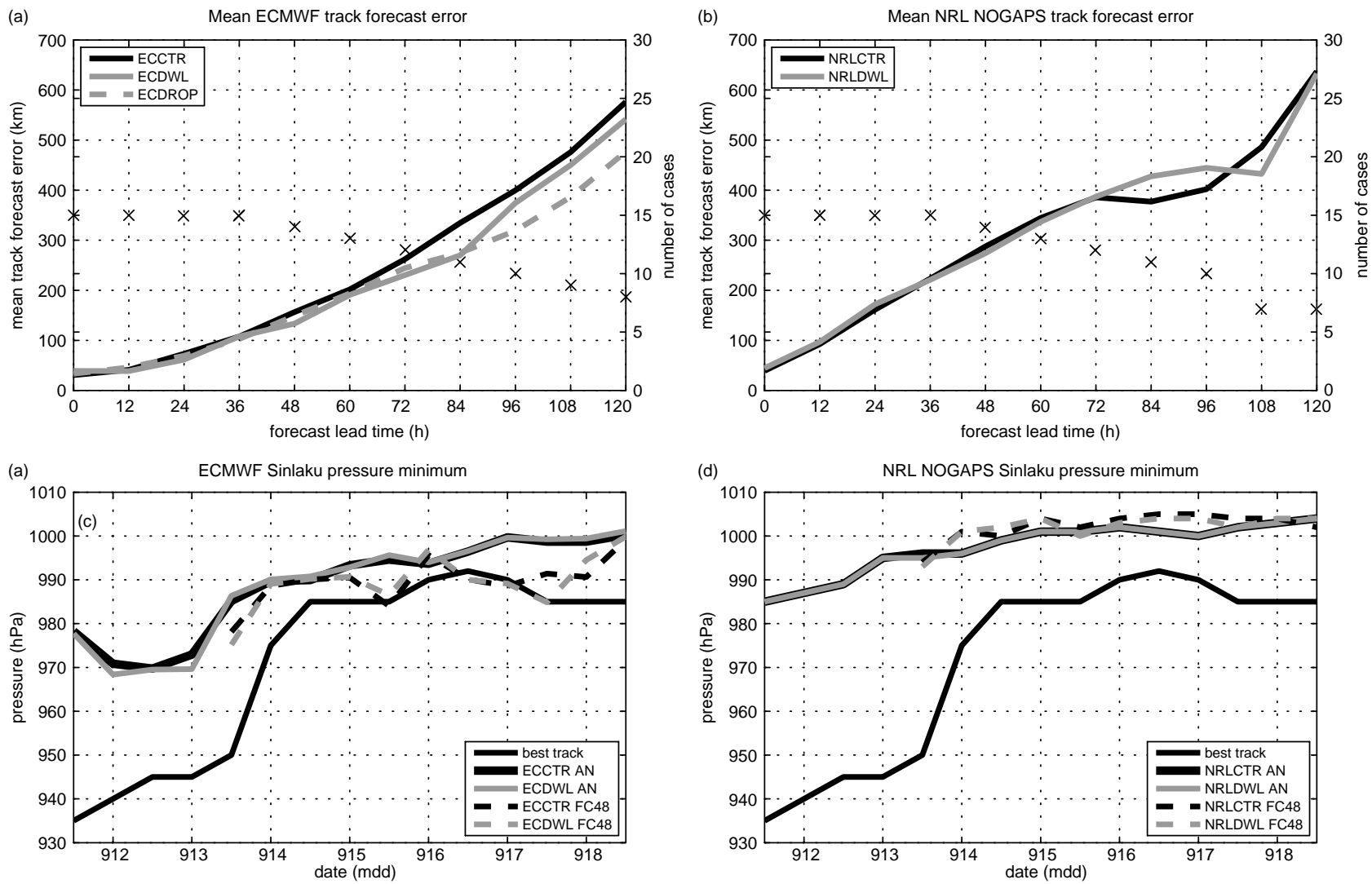

Figure 5. (a) ECMWF mean track forecast error of Sinlaku averaged over all forecasts initialized in the period 11-18 September and verified latest by 0000 UTC on 20 September. (b) Same as (a) but for NRL NOGAPS. (c) Sinlaku minimum pressure derived from best-track data (thin black solid line), ECCTR and ECDWL analysis fields (thick black and thin grey line) and 48h ECCTR and ECDWL forecasts (dashed black and grey line). (d) Same as (c), but for NRL NOGAPS analyses and forecasts. The number of averaged forecasts is shown with ' $x$ ' symbols and the right $y$-axis in (a) and (b).
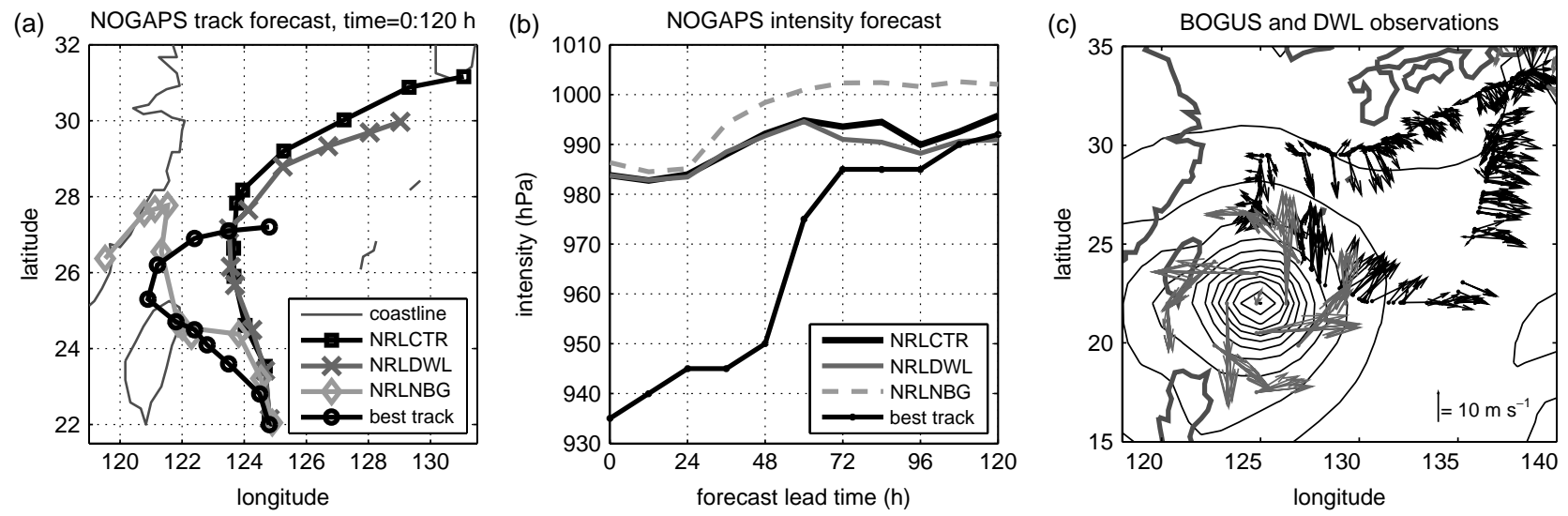

Figure 6. (a) NOGAPS Sinlaku track forecasts initialized at 1200 UTC on 11 September 2008, with markers indicating the typhoon position every 12 h. (b) Intensity forecasts corresponding to (a). (c) NRLDWL $1000 \mathrm{hPa}$ geopotential height first-guess field of the initial analysis in contours with bogus (grey arrows) and DWL (black arrows) observations on 11 September at all heights. A reference arrow for $10 \mathrm{~m} \mathrm{~s}^{-1}$ is located to the lower right.

campaign. Flight routes for the observations were partly guided by a range of different sensitive area calculations optimized for Typhoon Sinlaku and different verification boxes downstream over the Pacific. The observations are located in the environment of Typhoon Sinlaku, the interface between Sinlaku and the midlatitude jet and the jet stream itself. Wind profiles from coherent DWL are often intermittent due to the lack of sufficient scattering aerosols or the absorption of the signal by clouds. On average, every profile covers a vertical extent of $2500 \mathrm{~m}$.

Despite the differences of the two data assimilation and modelling systems, e.g. resolution, model dynamics, physical parameterization, observation operator and number and type of assimilated observations, the observation departures statistics are quite similar, with RMS innovations around $3 \mathrm{~m} \mathrm{~s}^{-1}$ and RMS residuals around $2 \mathrm{~m} \mathrm{~s}^{-1}$. DWL observations are volume averages and thus expected to have lower representativeness errors than other in situ observations (Weismann et al., 2005). They were assimilated with assigned error variances of $1.5 \mathrm{~m} \mathrm{~s}^{-1}$ at ECMWF, which is lower than the error of any other wind observation, and with $1.8 \mathrm{~m} \mathrm{~s}^{-1}$ in the NOGAPS system, which is equal to the most accurate other wind observations (radiosondes at low levels). The magnitude of the innovations and the very 

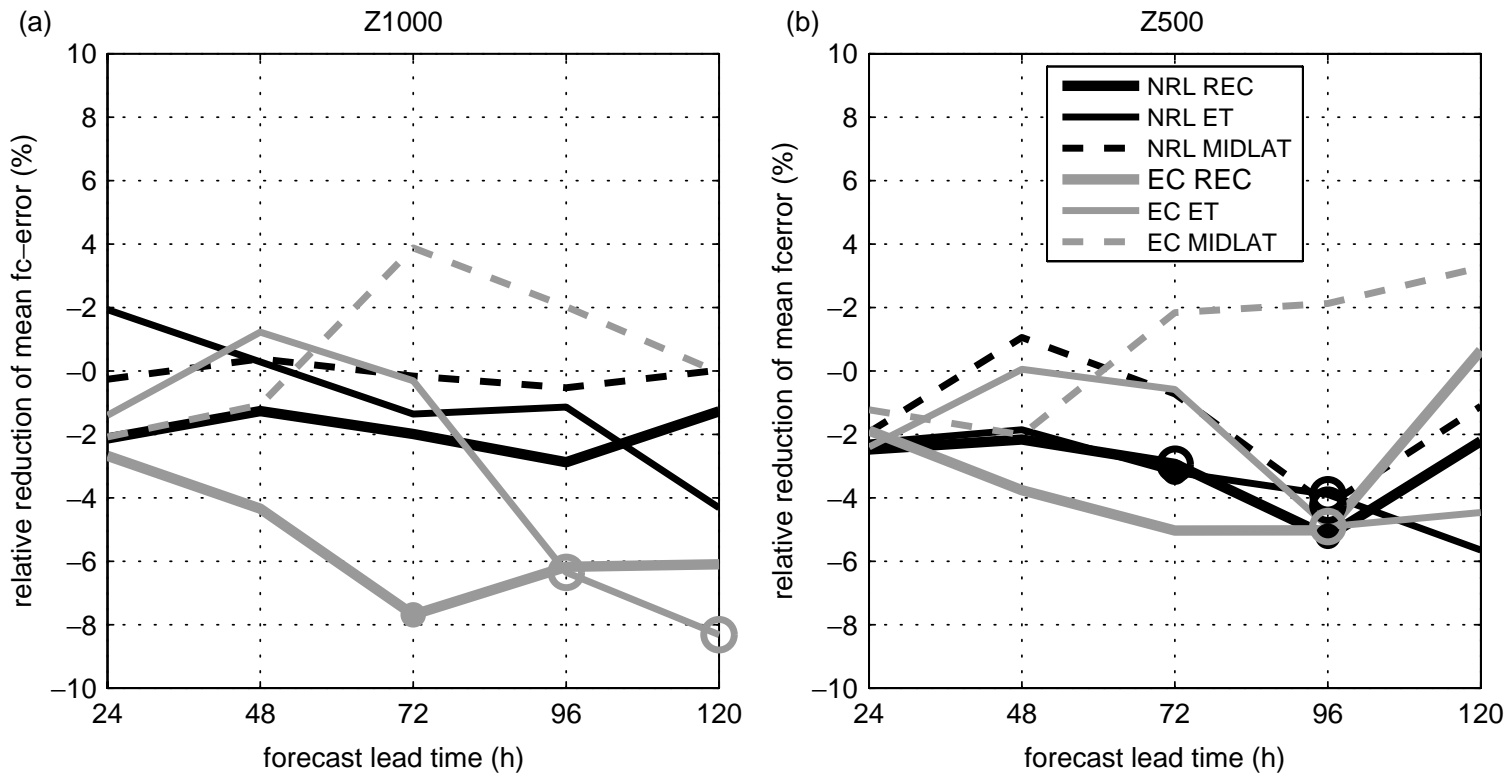

Figure 7. Relative reduction of mean geopotential height forecast errors with DWL observations compared to the control experiments without DWL at the (a) $1000 \mathrm{hPa}$ level and (b) $500 \mathrm{hPa}$ level in three different verification boxes (REC, ET and MIDLAT) shown in Figure 1. Negative values represent lower errors in the experiments with DWL observations. Differences that are statistically significant at a 90\% (95\%) confidence level are indicated with empty (filled) circles.

Table II. DWL and Sinlaku bogus observation impact on $24 \mathrm{~h}$ forecast error in experiment NRLDWL. Negative values stand for a reduction of forecast error, positive ones for an increase.

\begin{tabular}{lcccccrc}
\hline & $\begin{array}{c}\text { DWL error } \\
\text { reduction } \\
\left(\mathrm{J} \mathrm{kg}^{-1}\right)\end{array}$ & $\begin{array}{c}\text { DWL error } \\
\text { reduction per } \\
\text { observation } \\
\left(\times 10^{-5} \mathrm{~J} \mathrm{~kg}^{-1}\right)\end{array}$ & $\begin{array}{c}\text { DWL beneficial } \\
\text { percentage }\end{array}$ & $\begin{array}{c}\text { Number of } \\
\text { DWL }(u, v)\end{array}$ & $\begin{array}{c}\text { Bogus } \\
\text { beneficial } \\
\text { percentage }\end{array}$ & $\begin{array}{c}\text { Bogus } \\
\text { error } \\
\text { reduction } \\
\left(\mathrm{J} \mathrm{kg}^{-1}\right)\end{array}$ & $\begin{array}{c}\text { Number of } \\
\text { Sinlaku } \\
\text { bogus } \\
(u, v, T, Z)\end{array}$ \\
\hline 2008091106 & -0.028 & -6.8 & $54.7 \%$ & 418 & $41.7 \%$ & 0.028 & 139 \\
2008091112 & -0.006 & -1.3 & $49.8 \%$ & 470 & $50.0 \%$ & -0.038 & 138 \\
2008091400 & -0.014 & -2.8 & $42.3 \%$ & 496 & $52.7 \%$ & -0.006 & 131 \\
2008091406 & 0.003 & 1.8 & $44.7 \%$ & 188 & $41.0 \%$ & -0.002 & 122 \\
2008091600 & -0.006 & -1.4 & $49.2 \%$ & 388 & $52.3 \%$ & -0.006 & 130 \\
2008091606 & -0.002 & -0.7 & $55.6 \%$ & 270 & $53.8 \%$ & 0.002 & 130 \\
2008091706 & -0.004 & -1.1 & $49.0 \%$ & 360 & $53.4 \%$ & -0.017 & 161 \\
2008091712 & 0.004 & 4.6 & $45.0 \%$ & 80 & $57.5 \%$ & -0.018 & 134 \\
2008091806 & -0.013 & -2.0 & $55.6 \%$ & 664 & & & 0 \\
2008091900 & 0.004 & 1.4 & $53.2 \%$ & 316 & $52.5 \%$ & -0.012 & 118 \\
2008091906 & 0.004 & 1.6 & $48.2 \%$ & 224 & $55.7 \%$ & -0.013 & 122 \\
2008092100 & -0.032 & -6.4 & $58.7 \%$ & 494 & & & 0 \\
All & -0.09 & -2.1 & $51.4 \%$ & 4368 & $56.51 \%$ & -0.082 & 1325 \\
\hline
\end{tabular}

low number of discarded observations confirm their high accuracy and representativeness.

The higher weight and the larger number of DWL observations contribute to a larger mean analysis increment at the location of DWL observations in the ECMWF system compared to NAVDAS-AR. However, the mean absolute analysis and forecast difference with and without DWL observations averaged over the whole 11-day period and the area of the observations is slightly higher in the NOGAPS system. Likely this is due to the larger amount of satellite radiances assimilated at ECMWF that better constrain the analysis.

Overall, both models show a beneficial influence of DWL observations on the forecast skill. In the ECMWF system, the DWL observations lead to a mean relative reduction of the track forecast error of Typhoon Sinlaku by $9 \%$ for lead times of $12-120 \mathrm{~h}$. The improvement is comparable to the mean relative track error reduction in an experiment with 300 dropsondes. This means that DWL observations from one aircraft led to as much improvement as dropsondes from four aircraft in the same period, but it should be kept in mind that the investigation period is limited and this result cannot be generalized. In addition, a reduction of the mean 500 and $1000 \mathrm{hPa}$ geopotential height forecast error is seen in areas covering the track of Sinlaku and its environment, when forecasts are verified with an independent ECMWF analysis. The impact downstream over the Pacific in midlatitudes is negative, but the results are not significant.

The DWL influence on NOGAPS track forecasts of Sinlaku is relatively small. A significant issue with NOGAPS 
(a)

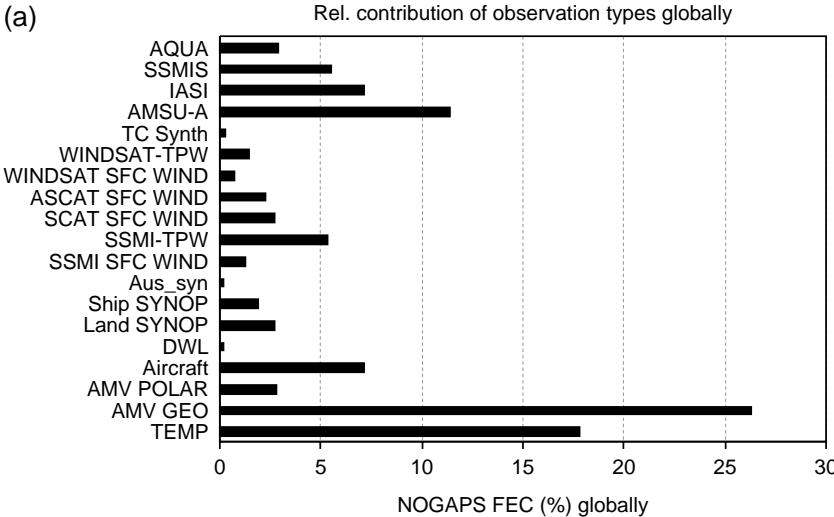

(c)

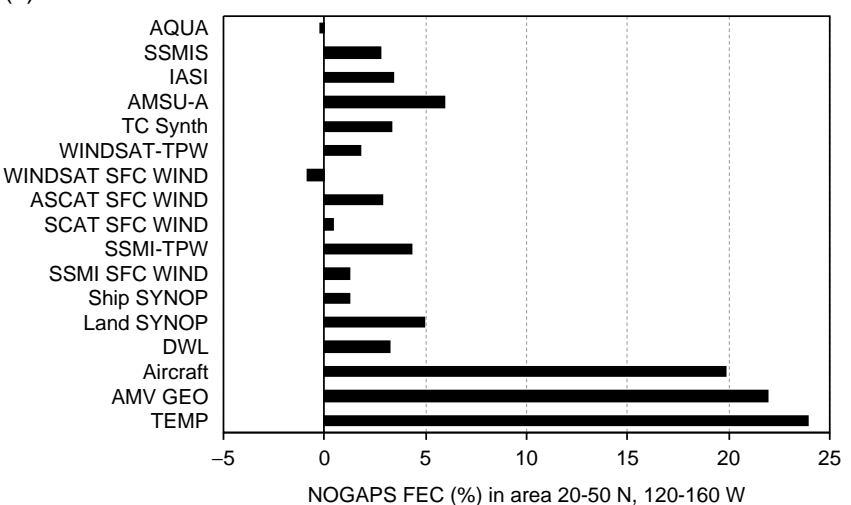

(b)

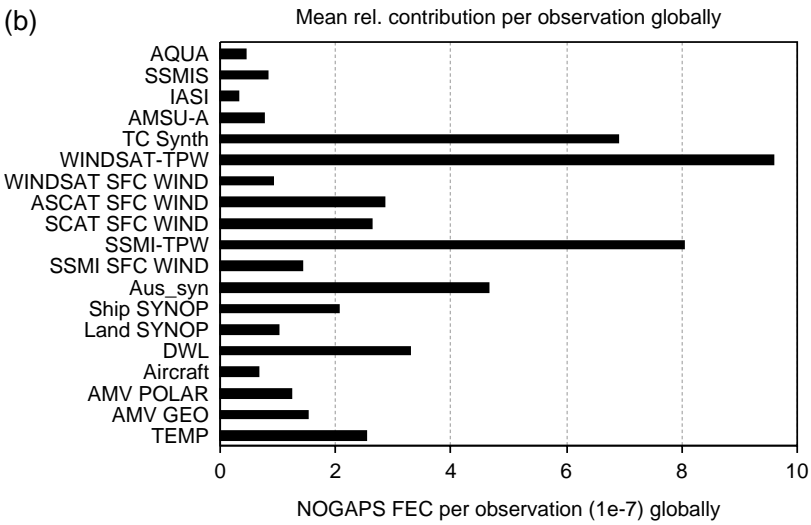

(d)

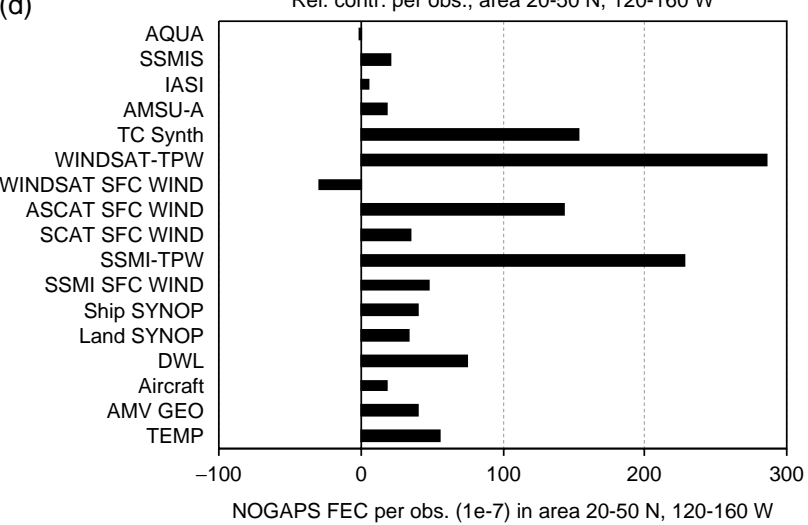

Figure 8. (a) Relative global contribution of observation types to the reduction of $24 \mathrm{~h}$ forecast error (computed in terms of a global moist total energy norm) in all 12 analysis cycles with DWL observations calculated with the NOGAPS adjoint forecast sensitivity to observations. (b) Mean relative global contribution per observation. (c) Relative contribution of observations in the area ET shown in Figure 1. (d) Mean relative contribution per observation in the area ET. Positive values represent a reduction of the forecast error by the observation type and negative values an increase. Note that the scaling of (a) and (c) is in percent, whereas the scaling of (b) and (d) is $10^{-7}$.

Table III. Calculated cumulative observation impact on $24 \mathrm{~h}$ forecast error in the experiments NRLDWL and NRLNBG for selected observation types at 1200 UTC on 11 September in a box around Sinlaku $\left(15-30^{\circ} \mathrm{N}\right.$ and $\left.117-132^{\circ} \mathrm{E}\right)$. Negative values stand for a reduction of forecast error, positive ones for an increase.

\begin{tabular}{lccc}
\hline & NRLDWL $\left(\mathrm{J} \mathrm{kg}^{-1}\right)$ & NRLNBG $\left(\mathrm{J} \mathrm{kg}^{-1}\right)$ & Difference $\left(\mathrm{J} \mathrm{kg}^{-1}\right)$ \\
\hline Bogus & -0.038 & 0.000 & 0.038 \\
Surface observations & -0.014 & -0.032 & -0.018 \\
Aircraft & 0.001 & 0.058 & 0.057 \\
AMV & -0.002 & -0.013 & -0.011 \\
SSMI surface wind speed & 0.006 & 0.007 & 0.001 \\
Scatterometer wind & -0.008 & -0.068 & -0.060 \\
DWL & -0.004 & -0.009 & -0.005 \\
Radiosondes & -0.008 & -0.031 & -0.023 \\
AMSU-A radiances & -0.005 & -0.007 & -0.002 \\
IASI and SSMI radiances & -0.003 & -0.012 & -0.009 \\
Sum, all observations & -0.075 & -0.107 & -0.032 \\
\hline
\end{tabular}

is that the synthetic tropical cyclone vortex bogus data substantially reduce the influence of additional observations, including DWL. However, given the fact that Sinlaku is systematically too weak in NOGAPS forecasts, these bogus data still appear to be necessary in the system. A sensitivity experiment without bogus, but with DWL observations in one analysis cycle, shows an improved track but also a weakening of the storm. Despite the overall limited impact on the typhoon forecasts, a consistent error reduction of NOGAPS geopotential height forecasts is visible in verification areas covering the track of Sinlaku and its environment. Furthermore, improvement of $500 \mathrm{hPa}$ geopotential height forecasts at lead time 4-5 days is seen downstream over the Pacific and differences are significant at 4 days lead time.

The Falcon T-PARC flights were partly guided by singular vector and ensemble transform Kalman-filter sensitive area calculations. Given the limited sample size, however, it was not possible to determine whether observations in sensitive regions had a higher influence than other observations. 
(a)

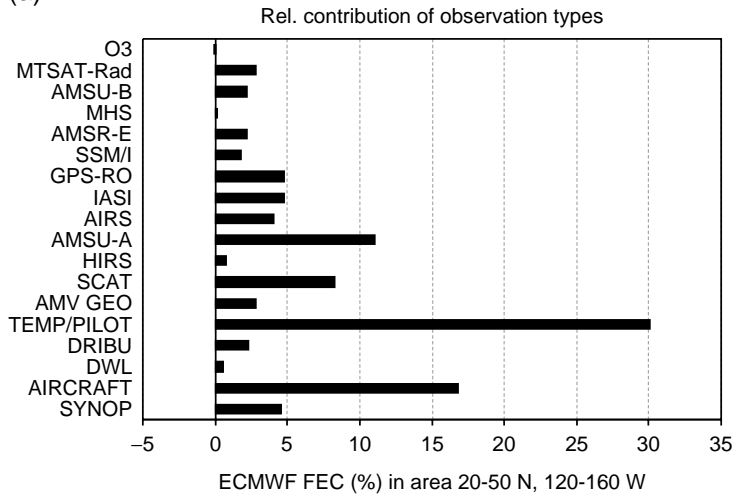

(b)

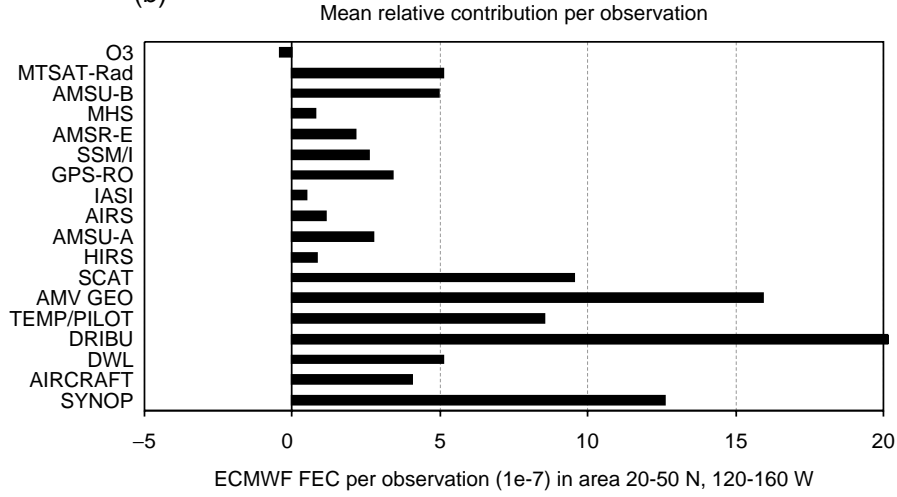

Figure 9. (a) Relative contribution of observation types in the area ET and the period 2008091100-2008092100 to the reduction of 24 h forecast error (computed in terms of a global dry total energy norm) calculated with the ECMWF adjoint forecast sensitivity to observations. (b) Mean relative contribution per observation in the area ET. The contribution of drifting buoys observations is $63 \times 10^{-7}$, which exceeds the scale. Note that the scaling of (a) is in percent, whereas the scaling of (b) is $10^{-7}$.

In both modelling systems, the influence of DWL observations on intensity forecasts is negligible. Presumably observations closer to the storm would be more beneficial. However, as DWL instruments cannot penetrate clouds, they are likely not the optimal observational platform for such phenomena.

In addition to data denial experiments, adjoint methods are used to quantify the impact of observations in the NOGAPS and ECMWF systems. The results confirm the beneficial influence of DWL observations. The total relative DWL contribution in the NOGAPS system is about twice as high as in the ECMWF system, which corresponds to a smaller analysis and forecast difference of $500 \mathrm{hPa}$ geopotential height fields with and without DWL in the ECMWF system. This may be related to the higher number of other observations assimilated in the ECMWF model, but also to the more sophisticated DWL assimilation procedure in NOGAPS that includes a superobbing scheme instead of a thinning procedure used in the ECMWF system. Another factor that likely increases the relative contribution of DWL observations in NOGAPS is the definition of the forecast error cost function, which has an upper limit of $150 \mathrm{hPA}$ in NOGAPS, in contrast to $0.1 \mathrm{hPa}$ in the ECMWF model.

In the NOGAPS system, the DWL impact per observation is higher than that of all other observation types, except synthetic observations, TPW satellite observations and ASCAT surface wind in the area near Typhoon Sinlaku. The contribution per DWL observations in the ECMWF system is similar to other aircraft observations, but smaller than drifting buoys, radiosonde and wind profiler observations, AMVs, SYNOP and scatterometer surface winds. This lower contribution per DWL observation may be related to the denser grid of DWL observations (i.e. higher number of DWL observations) assimilated in the ECMWF system, but also reflects the lower total impact of DWL observations in the ECMWF system.

Overall, the results confirm the high observation quality and the observation impact of airborne coherent DWL observations as documented by Weissmann and Cardinali (2007). It is possible to assimilate such observations without developing a completely new observation operator as the observations are not fundamentally different from conventional wind observations.

The impact of airborne DWL observations raises expectations for the first space-borne DWL instrument: the Atmospherics Dynamics Mission Aeolus (ADM-Aeolus) instrument that is planned to be launched by the European Space Agency in the near future (ESA, 2008). The ADMAeolus instrument will provide a global coverage of wind observations and it can also determine wind from molecular backscatter, which will provide wind information from regions with low aerosol content. It should be kept in mind that the errors of ADM-Aeolus with respect to airborne scanning DWL instruments will be roughly twice as high, the resolution will be lower and it will only provide the LOS wind information, i.e. one wind component. Nevertheless, the study documents that current NWP models are still lacking wind information in remote regions and DWL instruments are a promising source for providing such information in the future.

\section{Acknowledgements}

The DWL observations were collected as part of the Falcon operations during T-PARC, which were sponsored by an international consortium from the United States (National Science Foundation), Germany (DLR, Forschungszentrum Karlsruhe), Japan (JMA), Korea (National Institute of Meteorological Research) and Canada (Environment Canada). The role of the National Center for Atmospheric Research's Earth Observing Laboratory (NCAR EOL) for campaign and data management and the support from the US Navy for aircraft operations are acknowledged. The authors are thankful to all people who contributed to the successful realization of T-PARC and all operators of the DWL instrument. Furthermore, the support by Florian Harnisch, Anne Fouilloux and David Tan for ECMWF experiments is highly appreciated.

During most of the study, Martin Weissmann was part of the Deutsche Forschungsgemeinschaft (German Research Foundation) research unit PANDOWAE - Predictability and Dynamics of Weather Systems in the Atlantic-European Sector (www.pandowae.de). Since March 2011, Martin Weissmann has been part of the Hans-Ertel-Zentrum für Wetterforschung (http://www.dwd.de/forschung), funded by Deutscher Wetterdienst. The work of Rolf H. Langland and Patricia M. Pauley was supported by the Naval Research Laboratory through program element $0602435 \mathrm{~N}$, project number BE-435-046. 


\section{References}

Andersson E, Järvinen H. 1999. Variational quality control. Q. J. R. Meteorol. Soc. 125: 697-722.

Bormann N, Saarinen S, Kelly G, Thépaut J-N. 2003. The spatial structure of observation errors in atmospheric motion vectors from geostationary satellite data. Mon. Weather Rev. 131: 706-718.

Cardinali C. 2009. Monitoring the observation impact on the short-range forecast. Q. J. R. Meteorol. Soc. 135: 239-250.

Emanuel K, Kalnay E, Bishop C, Elsberry R, Gelaro R, Keyser D, Lord S, Rogers D, Shapiro M, Snyder C, Velden C. 1997. Observations in aid of weather prediction for North America: report of prospectus development team seven. Bull. Am. Meteorol. Soc. 78: 2859-2868.

ESA. 2008. 'ADM-Aeolus science report'. ESA Rep. SP-1311.

Gelaro R, Langland RH, Pellerin S, Todling R. 2010. The THORPEX observation impact inter-comparison experiment. Mon. Weather Rev. 138: 4009-4025.

Goerss JS, Jeffries RA. 1994. Assimilation of synthetic tropical cyclone observations into the Navy Operational Global Atmospheric Prediction System. Weather Forecast. 9: 557-576.

Grund CJ, Banta RM, George JL, Howell JN, Post MJ, Richter RA, Weickmann AM. 2001. High-resolution Doppler lidar for boundary layer and cloud research. J. Atmos. Oceanic Technol. 18: 376-393.

Harnisch F, Weissmann M. 2010. Sensitivity of typhoon forecasts to different subsets of targeted dropsonde observations. Mon. Weather Rev. 138: 2664-2680.

Harr PA, Sanabia ER, Penny AB. 2010. 'Typhoon Sinlaku during T-PARC: sensitivity of the re-intensification and downstream development to the track following recurvature'. In 29th Conference on Hurricanes and Tropical Meteorology, American Meteorological Society, Boston, MA. http://ams.confex.com/ams/pdfpapers/167999.pdf

Hogan T, Pauley RL. 2006. 'Sensitivity of tropical cyclone track forecasts to convective momentum transport in the NOGAPS Emanuel cumulus parameterization'. In 27th Conference on Hurricanes and Tropical Meteorology, Monterey, CA, USA. http://ams.confex.com/ams/pdfpapers/107309.pdf

Hogan TF, Rosmond TE. 1991. The description of the Navy Operational
Global Atmospheric System's spectral forecast model. Mon. Weather Rev. 119: 1786-1815

Isaksen L. 2010. 'The Operational Data Assimilation System'. ECMWF training course. http://www.ecmwf.int/newsevents/training/ meteorological_presentations/MET_DA.html

Langland RH, Baker NL. 2004. Estimation of observation impact using the NRL atmospheric variational data assimilation adjoint system. Tellus A 56: 189-201.

$\mathrm{Pu}$ Z, Zhang L, Emmitt GD. 2010. Impact of airborne Doppler wind lidar profiles on numerical simulations of a tropical cyclone. Geophys. Res. Lett. 37: L05801, DOI:10.1029/2009GL041765.

Rabier F, Järvinen H, Klinker E, Mahfouf JF, Simmons A. 2000. The ECMWF operational implementation of four-dimensional variational assimilation. Part 1: Experimental results with simplified physics. Q. J. R. Meteorol. Soc. 126: 1143-1170.

Rosmond T, Xu L. 2006. Development of NAVDAS-AR: non-linear formulation and outer loop tests. Tellus A 58: 45-58.

Smalikho I. 2003. Techniques of wind vector estimation from data measured with a scanning coherent Doppler lidar. J. Atmos. Oceanic Technol. 20: 276-291.

Stoffelen A, Pailleux J, Källén E, Vaughan JM, Isaksen L, Flamant P, Wergen W, Andersson E, Schyberg H, Culoma A, Meynart R, Endemann M, Ingmann P. 2005. The atmospheric dynamics mission for global wind measurement. Bull. Am. Meteorol. Soc. 86: $73-87$.

Velden CS, Bedka KM. 2009. Identifying the uncertainty in determining satellite-derived atmospheric motion vector height attribution. J. Appl. Meteorol. Climatol. 48: 450-463.

Weissmann M, Cardinali C. 2007. Impact of airborne Doppler lidar observations on ECMWF forecasts. Q. J. R. Meteorol. Soc. 133: $107-116$.

Weissmann M, Busen R, Dörnbrack A, Rahm S, Reitebuch O. 2005. Targeted observations with an airborne wind lidar. J. Atmos. Oceanic Technol. 22: 1706-1719.

Weissmann M, Harnisch F, Wu C-C, Lin P-H, Ohta Y, Yamashita K, Kim Y-H, Jeon E-H, Nakazawa T, Aberson S. 2011. The influence of assimilating dropsonde data on typhoon track and mid-latitude forecasts. Mon. Weather Rev. 139: 908-920. 